IJMMS 26:11 (2001) 685-694

PII. S0161171201005415

http://ijmms.hindawi.com

(c) Hindawi Publishing Corp.

\title{
REFLECTION AND TRANSMISSION OF ELASTIC WAVES AT VISCOUS LIQUID/MICROPOLAR ELASTIC SOLID INTERFACE
}

\author{
RAJNEESH KUMAR and SUSHIL K. TOMAR
}

(Received 16 March 1999)

\begin{abstract}
Amplitude ratios of various reflected and transmitted elastic waves from a plane interface separating a viscous liquid half-space and a micropolar elastic solid halfspace, are obtained in two cases (i) when longitudinal wave propagating through the solid half-space is made incident at the interface and (ii) when "coupled-wave" propagating through the solid half-space is made incident at the interface. These amplitude ratios have been computed numerically for a specific model and results obtained are presented graphically. It is found that these amplitude ratios depend on the angle of incidence of the incident wave and the effect of viscosity of the liquid on amplitude ratios is noticed. The problem studied by Tomar and Kumar (1995) has been reduced as a special case of our problem.
\end{abstract}

2000 Mathematics Subject Classification. 74F10, 74J20, 74B99.

1. Introduction. Classical theory of elasticity is not found adequate for material possessing granular structure. Particularly, the effect of granular structure or microstructure becomes important in the case of elastic vibrations of high frequency and/or small wavelength. Eringen and Suhubi [3, 4, 12] developed a theory in which they considered the microstructure of the material and they showed that the motion in a granular structured material is characterized not only by a displacement vector but also by a rotation vector. This theory is known as "theory of micropolar continuum."

In granular composites consisting of stiff inclusions embedded in a weaker matrix, a well-defined microstructure exists. Metal, polymers, composites, soils, rocks, concrete are typical media with microstructure. Most of the natural and man-made materials including engineering, geological and biological media posses a microstretch, in the usual environment they operate, become activated and influence dramatically the mechanical and other behaviours. Gautheir [6] found aluminum-epoxy composite to be a micropolar material and investigated the values of relevant parameters based on a specimen of an aluminum-epoxy composite. Gautheir and Jahsman [7] presented a quest for micropolar elastic constants and suggested that a material with nearly spherical and uniformly graded particulate could be expected to exhibit improved micropolar behaviour and micropolar effect that will become prominent only for extremely short wavelength phenomenon.

The problems of reflection and refraction of plane waves at plane interface have been studied extensively by many authors (see Brekhovskikh [2]). Miles [9] studied the problem of dispersive reflection at the interface between ideal and viscous liquid 
media. Interaction of seismic waves with a viscous liquid layer has been investigated by Fehler [5]. Parfitt and Eringen [10], Ariman [1], Tomar and Gogna [13], Tomar and Kumar [8, 14], and Singh and Kumar [11] discussed some problems on reflection and refraction in a micropolar media. Although the work at the interface between microscopic and macroscopic scales has a long history, the field has become particularly active in recent years. This activity has been motivated, in the past, by the need for increasing detailed models of classical phenomenon such as dispersion of weak elastic waves and, in part, by the requirement for development of comprehensive, physically motivated theories of behaviour of continuous media. Problems of the former sort have motivated development of theories of micropolar elasticity, methods of evaluating statistical properties of the response of randomly inhomogeneous media, and so forth. Development of increasingly comprehensive continuum theories, always a topic of interest, has increased its practical importance in recent years in response to the requirement for explaining the phenomenon lying outside the range of the more conventional continuum models, and because advances in computational mechanics have made the wide-spread application of these theories possible. Much of the development of comprehensive modern constitutive theories has occurred through interaction of mechanicians with persons analyzing finer scale continuum behaviour and with material scientists and others outside the field of mechanics. This has imposed a requirement for inter-disciplinary communication that motivates most of the work at the micro/macro interface. When a scientist or engineer, solving problems at the macroscopic level wants to take advantage of the knowledge of microscopic processes, it is appropriate to adopt or develop a theory that incorporates these processes in some averaged, but explicit fashion.

Micropolar theory of elasticity was developed in the sixties by Eringen and Suhubi $[3,4,12]$ and the availability of experimental data for various parameters of micropolar material gives us a chance to consider such problems of reflection and refraction where aluminum-epoxy composites as micropolar elastic solid is in welded contact with the crust as a viscous liquid half-space. Such a model may be found in the earth's crust, and the results of this problem can be applicable to the earth's crust, to a watermud-rock boundary, or to some other specific problem in engineering seismology like bedrock-soil interface or mantle-crust interface. In the present investigation, we consider the problem of reflection and transmission of plane elastic wave (longitudinal wave or coupled-wave) at an interface separating the micropolar elastic solid and the viscous liquid half-spaces.

2. Basic equations and constitutive relations. The equations of motion in viscous liquid medium are given by [5]

$$
K^{\prime} \nabla^{2} \phi^{\prime}+\frac{4}{3} \eta \frac{\partial}{\partial t} \nabla^{2} \phi^{\prime}=\rho^{\prime} \frac{\partial^{2} \phi^{\prime}}{\partial t^{2}}, \quad \eta \frac{\partial}{\partial t} \nabla^{2} \psi^{\prime}=\rho^{\prime} \frac{\partial^{2} \psi^{\prime}}{\partial t^{2}}
$$

where $K^{\prime}$ is the bulk modulus, $\rho^{\prime}$ is the fluid density, $\eta$ is the fluid viscosity, $\phi^{\prime}$ and $\psi^{\prime}$ are the potentials corresponding to longitudinal and transverse waves, respectively. 
The components of displacement and stresses are given by

$$
\begin{gathered}
u^{\prime}=\frac{\partial \phi^{\prime}}{\partial x}-\frac{\partial \psi^{\prime}}{\partial z}, \quad w^{\prime}=\frac{\partial \phi^{\prime}}{\partial z}+\frac{\partial \psi^{\prime}}{\partial x}, \quad t_{z x}^{\prime}=\eta \frac{\partial}{\partial t}\left[2 \frac{\partial^{2} \phi^{\prime}}{\partial x \partial z}+\frac{\partial^{2} \psi^{\prime}}{\partial x^{2}}-\frac{\partial^{2} \psi^{\prime}}{\partial z^{2}}\right] \\
t_{z z}^{\prime}=\left[K^{\prime}-\frac{2}{3} \eta \frac{\partial}{\partial t}\right]\left[\frac{\partial^{2} \phi^{\prime}}{\partial x^{2}}+\frac{\partial^{2} \phi^{\prime}}{\partial z^{2}}\right]+2 \eta \frac{\partial}{\partial t}\left[\frac{\partial^{2} \psi^{\prime}}{\partial x \partial z}-\frac{\partial^{2} \phi^{\prime}}{\partial z^{2}}\right] .
\end{gathered}
$$

The equations of motion in micropolar elastic solid medium are given by [3]

$$
\begin{gathered}
\left(c_{1}^{2}+c_{3}^{2}\right) \nabla^{2} \phi=\frac{\partial^{2} \phi}{\partial t^{2}}, \\
\left(c_{2}^{2}+c_{3}^{2}\right) \nabla^{2} \vec{U}+c_{3}^{2} \nabla \times \vec{\Phi}=\frac{\partial^{2} \vec{U}}{\partial t^{2}}, \\
{\left[c_{4}^{2} \nabla^{2}-2 \omega_{0}^{2}\right] \vec{\Phi}+\omega_{0}^{2} \nabla \times \vec{U}=\frac{\partial^{2} \vec{\Phi}}{\partial t^{2}},}
\end{gathered}
$$

where

$$
c_{1}^{2}=\frac{\lambda+2 \mu}{\rho}, \quad c_{2}^{2}=\frac{\mu}{\rho}, \quad c_{3}^{2}=\frac{K}{\rho}, \quad c_{4}^{2}=\frac{\gamma}{\rho j}, \quad \omega_{0}^{2}=\frac{K}{\rho j} .
$$

Parfitt and Eringen [10] have shown that (2.3a) corresponds to longitudinal wave propagating with velocity $V_{1}$, given by $V_{1}^{2}=c_{1}^{2}+c_{3}^{2}$, and (2.3b), (2.3c) are coupled equations in vector potentials $\vec{U}$ and $\vec{\Phi}$ and these correspond to coupled transverse and micro-rotation waves. If $\omega^{2} / \omega_{0}^{2}>2$, there exist two sets of coupled-wave propagating with velocities $1 / \lambda_{1}$ and $1 / \lambda_{2}$; where

$$
\lambda_{1,2}^{2}=\frac{1}{2}\left[B \mp \sqrt{B^{2}-4 C}\right],
$$

where

$$
B=\frac{q(p-2)}{\omega^{2}}+\frac{1}{\left(c_{2}^{2}+c_{3}^{2}\right)}+\frac{1}{c_{4}^{2}}, \quad C=\left(\frac{1}{c_{4}^{2}}-\frac{2 q}{\omega^{2}}\right) \frac{1}{\left(c_{2}^{2}+c_{3}^{2}\right)}, \quad p=\frac{K}{\mu+K}, q=\frac{K}{\gamma} .
$$

We consider a two-dimensional problem by taking the following components of displacement and microrotation as

$$
\vec{u}=(u, 0, w), \quad \vec{\Phi}=\left(0, \Phi_{2}, 0\right),
$$

where

$$
u=\frac{\partial \phi}{\partial x}-\frac{\partial \psi}{\partial z}, \quad w=\frac{\partial \phi}{\partial z}+\frac{\partial \psi}{\partial x}
$$

and components of stresses as

$$
\begin{aligned}
& t_{z z}=(\lambda+2 \mu+K) \frac{\partial^{2} \phi}{\partial z^{2}}+\lambda \frac{\partial^{2} \phi}{\partial x^{2}}+(2 \mu+K) \frac{\partial^{2} \psi}{\partial x \partial z}, \\
& t_{z x}=(2 \mu+K) \frac{\partial^{2} \phi}{\partial x \partial z}-(\mu+K) \frac{\partial^{2} \psi}{\partial z^{2}}+\mu \frac{\partial^{2} \psi}{\partial x^{2}}-K \phi_{2}, \quad m_{z y}=\gamma \frac{\partial \phi_{2}}{\partial z} .
\end{aligned}
$$

3. Formulation of the problem and its solution. We consider a two-dimensional problem by taking the $z$-axis pointing into the lower half-space and the plane interface 


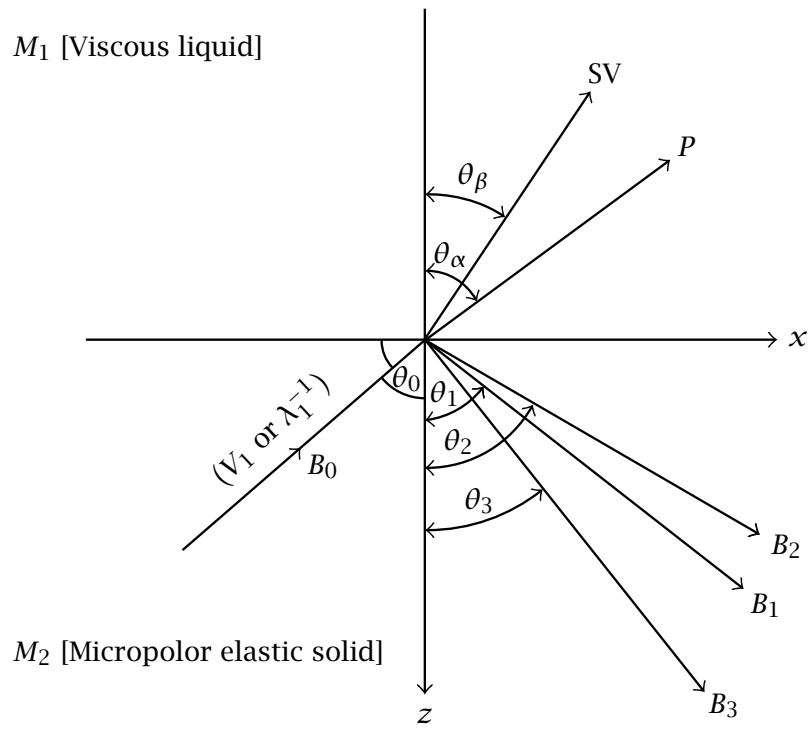

FIGURE 3.1. Geometry of the problem: reflection and refraction of waves.

$z=0$ separating the viscous liquid half-space $M_{1}[z<0]$ and uniform micropolar elastic solid half-space $M_{2}[z>0]$ (see Figure 3.1).

We take the following form of potentials in the two media.

In medium $M_{1}$ (see [9])

$$
\begin{aligned}
& \phi^{\prime}=A_{1} \exp \left\{-\iota\left(K^{\prime 2}-k_{\alpha}^{2} \sin ^{2} \theta_{\alpha}\right)^{1 / 2} z\right\} \exp \left\{\iota\left(\omega_{\alpha} t+k_{\alpha} x \sin \theta_{\alpha}\right)\right\}, \\
& \psi^{\prime}=A_{2} \exp \left\{-\iota\left(\frac{\iota \omega}{v}+k_{\beta}^{2} \sin ^{2} \theta_{\beta}\right)^{1 / 2} z\right\} \exp \left\{\iota\left(\omega_{\beta} t+k_{\beta} x \sin \theta_{\beta}\right)\right\},
\end{aligned}
$$

where $A_{1}, A_{2}$ are unknown to be determined from the boundary conditions and

$$
K^{\prime}=\frac{\omega}{c^{\prime}}\left(1+\frac{4}{3} \frac{\iota \omega v}{c^{\prime 2}}\right)^{-1 / 2}, \quad c^{\prime 2}=\frac{K^{\prime}}{\rho^{\prime}}, \quad v=\frac{\eta}{\rho^{\prime}},
$$

where $k_{\alpha}$ and $k_{\beta}$ are wave numbers of refracted $P$ - and SV-waves, respectively.

In medium $M_{2}$ (see [10])

$$
\begin{aligned}
\phi= & B_{0} \exp \left\{\iota k_{0}\left(\sin \theta_{0} x-\cos \theta_{0} z\right)+\iota \omega_{1} t\right\} \\
& +B_{1} \exp \left\{\iota k_{0}\left(\sin \theta_{1} x+\cos \theta_{1} z\right)+\iota \omega_{1} t\right\}, \\
\psi= & B_{0} \exp \left\{\iota \delta_{1}\left(\sin \theta_{0} x-\cos \theta_{0} z\right)+\iota \omega_{2} t\right\} \\
& +B_{2} \exp \left\{\iota \delta_{1}\left(\sin \theta_{2} x+\cos \theta_{2} z\right)+\iota \omega_{2} t\right\} \\
& +B_{3} \exp \left\{\iota \delta_{2}\left(\sin \theta_{3} x+\cos \theta_{3} z\right)+\iota \omega_{2} t\right\}, \\
\phi_{2}= & E B_{0} \exp \left\{\iota \delta_{1}\left(\sin \theta_{0} x-\cos \theta_{0} z\right)+\iota \omega_{3} t\right\} \\
& +E B_{2} \exp \left\{\iota \delta_{1}\left(\sin \theta_{2} x+\cos \theta_{2} z\right)+\iota \omega_{3} t\right\} \\
& +F B_{3} \exp \left\{\iota \delta_{2}\left(\sin \theta_{3} x+\cos \theta_{3} z\right)+\iota \omega_{3} t\right\},
\end{aligned}
$$


where $B_{0}, B_{1}, B_{2}$, and $B_{3}$ are unknown and

$$
\begin{gathered}
E=\frac{\delta_{1}^{2}\left(\delta_{1}^{2}-\omega^{2} /\left(c_{2}^{2}+c_{3}^{2}\right)+p q\right)}{\operatorname{deno}}, \\
F=\frac{\delta_{2}^{2}\left(\delta_{2}^{2}-\omega^{2} /\left(c_{2}^{2}+c_{3}^{2}\right)+p q\right)}{\operatorname{deno}}, \quad \operatorname{deno}=p\left(2 q-\frac{\omega^{2}}{c_{4}^{2}}\right), \delta_{1}^{2}=\lambda_{1}^{2} \omega^{2}, \delta_{2}^{2}=\lambda_{2}^{2} \omega^{2} .
\end{gathered}
$$

3.1. Boundary conditions. The appropriate boundary conditions are the continuity of displacement, microrotation and stresses at the interface separating media $M_{1}$ and $M_{2}$. Mathematically, these boundary conditions can be expressed as:

at $z=0$,

$$
t_{z z}=t_{z z}^{\prime}, \quad t_{z x}=t_{z x}^{\prime}, \quad m_{z y}=0, \quad u=u^{\prime}, \quad w=w^{\prime} .
$$

3.2. Reflection and refraction. For incident longitudinal wave at an interface $z=0$, we put $B_{0}=0$ in (3.3b) and (3.3c) and for incident coupled-wave we put $B_{0}=0$ in (3.3a) and (3.3c). Substituting the expressions of potentials given by (3.1) and (3.3) in the boundary conditions (3.5) and making use of Snell's law, given by

$$
\frac{\sin \theta_{0}}{V_{i}}=\frac{\sin \theta_{1}}{V_{i}}=\frac{\sin \theta_{2}}{\lambda_{1}^{-1}}=\frac{\sin \theta_{3}}{\lambda_{2}^{-1}}=\frac{\sin \theta_{\alpha}}{c_{p}^{\prime}}=\frac{\sin \theta_{\beta}}{c_{s}^{\prime}},
$$

where

$$
\begin{aligned}
& V_{i}= \begin{cases}V_{1}, & \text { for incident longitudinal wave, } \\
\lambda_{1}^{-1}, & \text { for incident coupled-wave, }\end{cases} \\
& c_{p}^{\prime}=\left[\frac{K^{\prime}}{\rho^{\prime}}\left(1+\frac{4}{3} \frac{\iota \omega \eta}{K^{\prime}}\right)\right]^{1 / 2}, \quad c_{s}^{\prime}=\left[\frac{\iota \omega \eta}{\rho^{\prime}}\right]^{1 / 2} .
\end{aligned}
$$

Also, frequencies of all the waves must be equal at the interface $z=0$ for all positions and time. Hence, we obtain a system of five equations which in matrix form are

$$
\begin{gathered}
A Z=B, \\
Z=\left[\begin{array}{lllll}
Z_{1} & Z_{2} & Z_{3} & Z_{4} & Z_{5}
\end{array}\right]^{t}, \\
Z_{1}=\frac{B_{1}}{B_{0}}, \quad Z_{2}=\frac{B_{2}}{B_{0}}, \quad Z_{3}=\frac{B_{3}}{B_{0}}, \quad Z_{4}=\frac{A_{1}}{B_{0}}, \quad Z_{5}=\frac{A_{2}}{B_{0}},
\end{gathered}
$$

where $Z_{1}$ to $Z_{5}$ are the amplitude ratios of reflected longitudinal wave, reflected coupled-wave at an angle $\theta_{2}$, reflected coupled-wave at an angle $\theta_{3}$, refracted $P$-wave and refracted SV-wave, respectively.

The elements of matrix $A$ in nondimensional form can be written as

$$
\begin{aligned}
& a_{11}=\left[\frac{\lambda}{\mu}+D_{2} \cos ^{2} \theta_{1}\right] \frac{k_{0}^{2}}{k^{* 2}}, \\
& a_{12}=D_{2} \sin \theta_{2} \cos \theta_{2} \frac{\delta^{2}}{k^{* 2}},
\end{aligned}
$$




$$
\begin{aligned}
& a_{13}=D_{2} \sin \theta_{3} \cos \theta_{3} \frac{\delta^{2}}{k^{* 2}}, \\
& a_{14}=-\frac{k^{2}}{k^{* 2}}\left[\frac{K^{\prime}}{\mu}-\frac{2}{3} \frac{\iota \omega \eta}{\mu}\right]+\frac{2 \eta \iota \omega}{\mu}\left[\frac{k^{2}}{k^{* 2}}-\sin ^{2} \theta\right], \\
& a_{15}=\frac{2 \eta \omega \sin \theta_{0}}{\mu}\left[\frac{\iota \omega}{v} k^{* 2}+\sin ^{2} \theta_{0}\right]^{1 / 2} \text {, } \\
& a_{21}=D_{2} \sin \theta_{1} \cos \theta_{1} \frac{k_{0}^{2}}{k^{* 2}}, \quad a_{31}=\frac{k_{0}}{k^{*}} \sin \theta_{1}, \\
& a_{22}=-\frac{\delta_{1}^{2}}{k^{* 2}}\left[\left(D_{1} \cos ^{2} \theta_{2}-\sin ^{2} \theta_{2}\right)-\frac{K}{\mu} \frac{E}{\delta_{1}^{2}}\right], \quad a_{32}=-\frac{\delta_{1}}{k^{*}} \cos \theta_{2}, \\
& a_{23}=-\frac{\delta_{2}^{2}}{k^{* 2}}\left[\left(D_{1} \cos ^{2} \theta_{3}-\sin ^{2} \theta_{3}\right)-\frac{K}{\mu} \frac{F}{\delta_{2}^{2}}\right], \quad a_{33}=-\frac{\delta_{2}}{k^{*}} \cos \theta_{3}, \\
& a_{24}=\frac{2 \iota k_{\alpha} \omega \eta}{\mu k^{* 2}}\left[\sin \theta_{\alpha}\left(1-\frac{k_{\alpha}^{2} \sin ^{2} \theta_{\alpha}}{k^{* 2}}\right)^{1 / 2}\right], \quad a_{34}=-\sin \theta_{0}, \\
& a_{25}=-\frac{\iota \omega \eta}{\mu}\left[2 \sin ^{2} \theta_{0}+\frac{\iota \omega}{v k^{* 2}}\right], \quad a_{35}=\left[\frac{\iota \omega}{v k^{* 2}}+\sin ^{2} \theta_{0}\right]^{1 / 2}, \\
& \begin{aligned}
a_{41} & =\frac{k_{0}}{k^{*}} \cos \theta_{1}, & a_{51} & =0, \\
a_{42} & =\frac{\delta_{1}}{k^{*}} \sin \theta_{2}, & a_{52} & =\cos \theta_{2}, \\
a_{43} & =\frac{\delta_{2}}{k^{*}} \sin \theta_{3}, & a_{53} & =\frac{\delta_{2} F}{\delta_{1} E} \cos \theta_{3}, \\
a_{44} & =\left[\frac{k^{2}}{k^{* 2}}-\sin ^{2} \theta_{0}\right]^{1 / 2}, & a_{54} & =a_{55}=0, \\
a_{45} & =-\sin \theta_{0}, & &
\end{aligned}
\end{aligned}
$$

where

$$
D_{1}=1+K, \quad D_{2}=1+D_{1}, \quad B=\left[\begin{array}{lllll}
b_{1} & b_{2} & b_{3} & b_{4} & b_{5}
\end{array}\right]^{t},
$$

(a) for incident longitudinal wave

$$
b_{1}=-a_{11}, \quad b_{2}=a_{21}, \quad b_{3}=-a_{31}, \quad b_{4}=a_{41}, \quad b_{5}=a_{51},
$$

(b) for incident coupled-wave

$$
\begin{gathered}
b_{1}=a_{12}, \quad b_{2}=-a_{22}, \quad b_{3}=a_{32}, \quad b_{4}=-a_{42}, \quad b_{5}=a_{52}, \\
k^{*}= \begin{cases}k_{0}, & \text { for incident longitudinal wave, } \\
\delta_{1}, & \text { for incident coupled-wave. }\end{cases}
\end{gathered}
$$


3.3. Special case. It can be easily verified that neglecting the viscous effect of the liquid, that is, putting $\eta=0$ in the boundary conditions given by (3.8a), one can obtain the same equations as obtained by Tomar and Kumar [14] in their problem of reflection and refraction at inviscid liquid/micropolar elastic solid interface where longitudinal displacement wave is made incident.

4. Numerical results. The theoretical results obtained above indicate that the amplitude ratios $Z_{i}(i=1,2,3,4,5)$ depend on the angle of incidence of incident wave. In order to study in more detail the behaviour of various amplitude ratios on the angle of incidence, we have computed them numerically by taking the following values of relevant elastic parameters.

In medium $M_{2}$,

$$
\begin{aligned}
& \lambda=7.59 \times 10^{11} \mathrm{dyne} / \mathrm{cm}^{2}, \quad \mu=1.89 \times 10^{11} \mathrm{dyne} / \mathrm{cm}^{2}, \\
& K=0.0149 \times 10^{11} \mathrm{dyne} / \mathrm{cm}^{2}, \quad \gamma=0.268 \times 10^{11} \mathrm{dyne}, \\
& \rho=2.19 \mathrm{~g} / \mathrm{cm}^{3}, \quad j=0.196 \mathrm{~cm}^{2}, \quad \frac{\omega^{2}}{\omega_{0}^{2}}=10 .
\end{aligned}
$$

In medium $M_{1}$,

$$
k^{\prime}=0.119 \times 10^{11} \text { dyne } / \mathrm{cm}^{2}, \quad \rho^{\prime}=1.01 \mathrm{~g} / \mathrm{cm}^{3}, \quad \eta=0.0014 \text { poise, }
$$

with these values of constants, we have solved the system of equations given by (3.8a) for different values of angle of incidence from 0 to 90 degrees.

Figures $4.1,4.2,4.3$, and 4.4 show the variation and effect of viscosity on amplitude ratios with angle of incidence. The modulus of amplitude ratios is represented by $Z_{i}$ in the case when the liquid is viscous while $Z_{i}{ }^{*}$ represents the amplitude ratios in the case when the liquid is inviscid.

Figure 4.1 shows the variation of amplitude ratios $Z_{i}$ and $Z_{i}{ }^{*}$ in the case when longitudinal wave is made incident at the interface. The graphs of $Z_{1}{ }^{*}, Z_{2}{ }^{*}$, and $Z_{3}{ }^{*}$ are depicted by magnifying 10,10 , and 100 times their original values, respectively. The variation of the rest of the amplitude ratios in this case is shown in Figure 4.2. In the case when coupled-wave is made incident at the interface, the variation of amplitude ratios $Z_{i}$ and $Z_{i}{ }^{*}$ is shown in Figure 4.3.

The variations of modulus of amplitude ratios $Z_{4}, Z_{5}$, and $Z_{4}^{*}$ have been depicted in Figure 4.4 .

5. Conclusions. In conclusion, a mathematical study of reflection and refraction coefficients at an interface separating the viscous-liquid and micropolar solid halfspaces is made when longitudinal wave is incident and when coupled-wave is incident at the interface. It is observed that (i) the amplitude ratios of various reflected and refracted waves depend on the angle of incidence of incident wave (ii) the effect of viscosity of the liquid on reflection and transmission coefficients is significant and the values of various amplitude ratios increase with the increase in the value of viscosity 


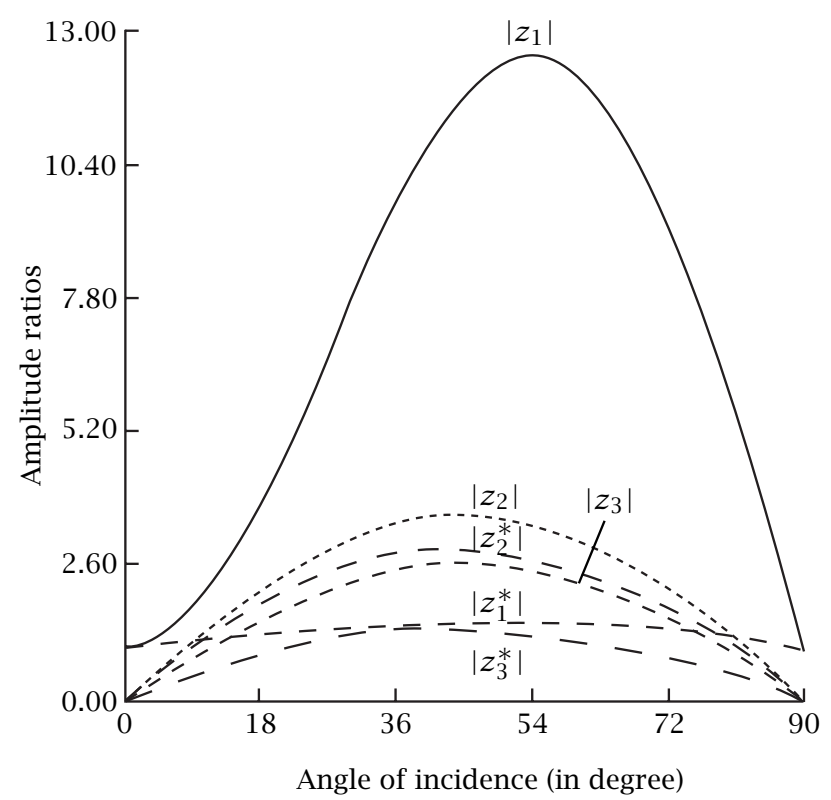

FIGURE 4.1. Variation of amplitude ratios with angle of incidence of longitudinal wave.

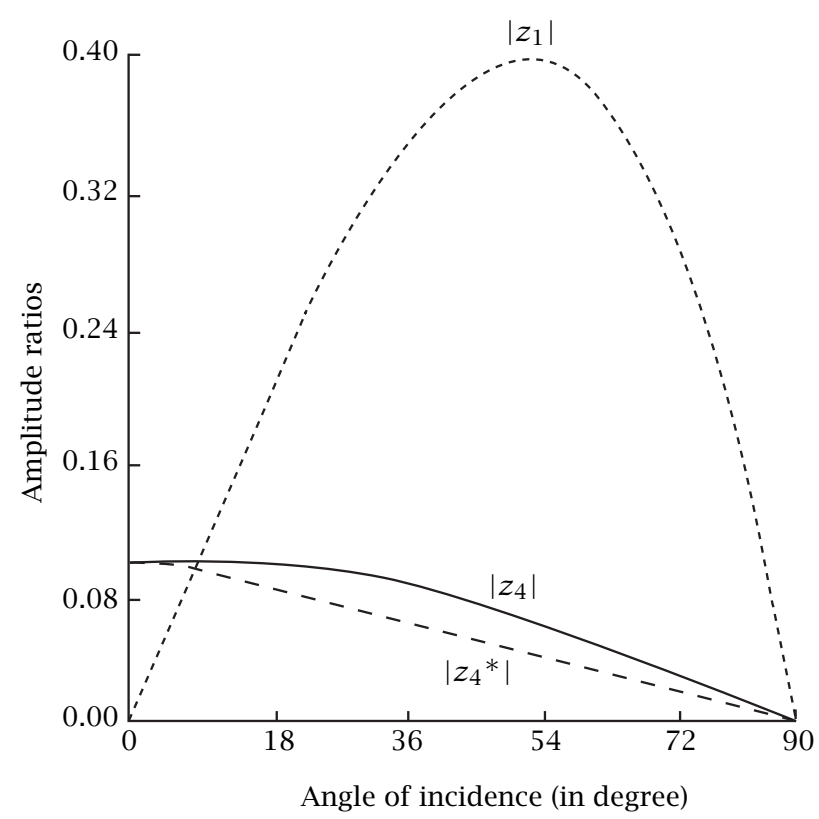

FIGURE 4.2. Variation of amplitude ratios with angle of incidence of longitudinal wave.

in the case when longitudinal wave is incident, while the value of amplitude ratio corresponding to reflected longitudinal wave decreases in case when coupled-wave is 


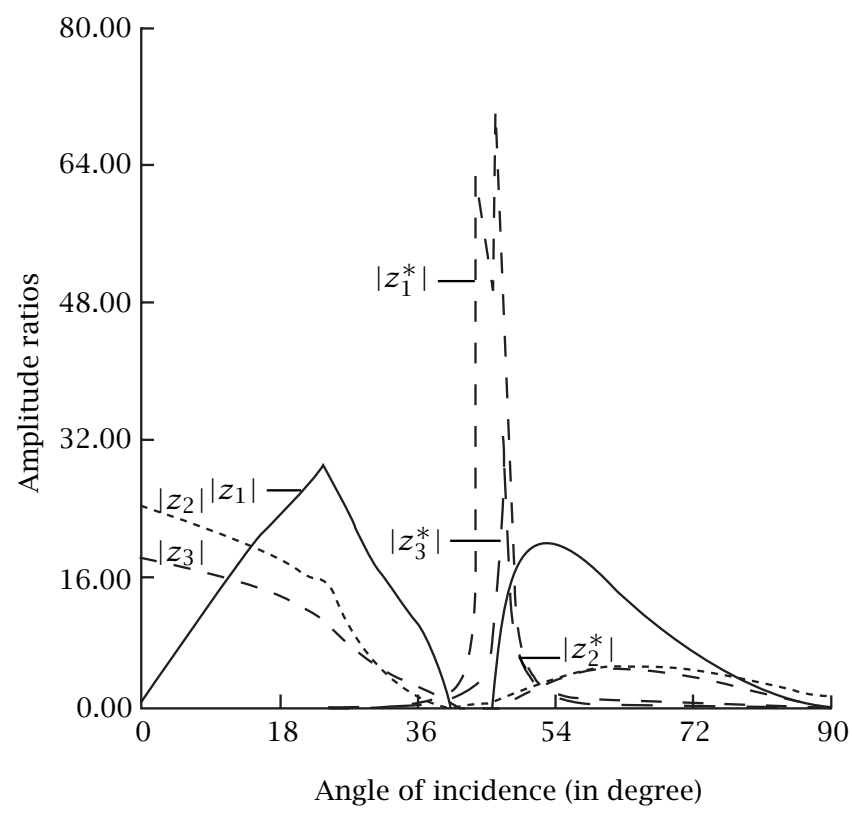

FIGURE 4.3. Variation of amplitude ratios with angle of incidence of coupled-wave.

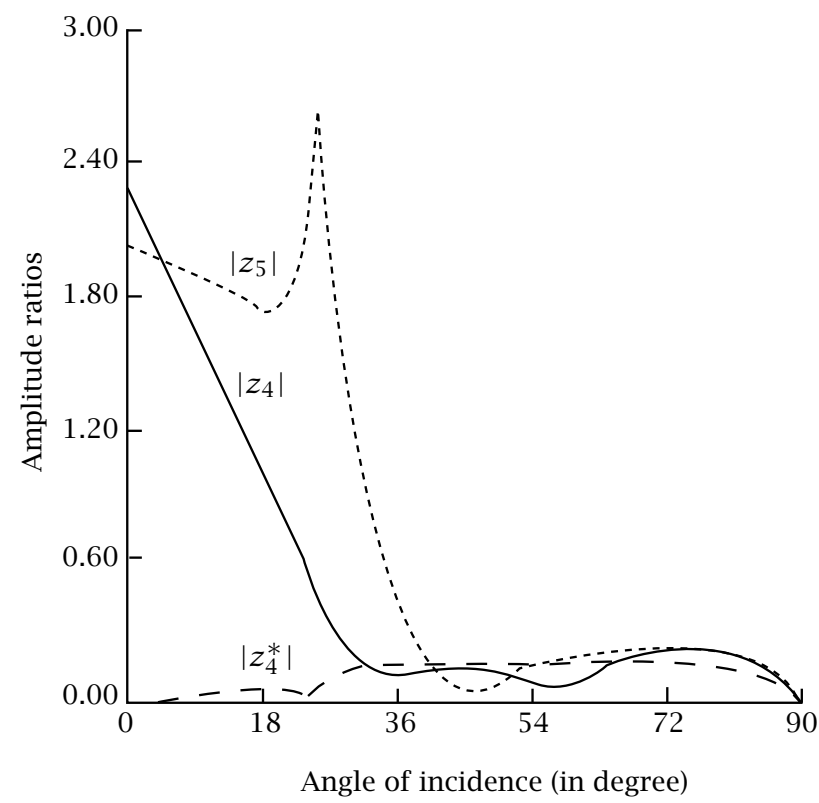

FIGURE 4.4. Variation of amplitude ratios with angle of incidence of coupled-wave.

incident. In the latter case, the values of the remaining amplitude ratios increase with the increase in the value of viscosity of the liquid. 


\section{REFERENCES}

[1] T. Ariman, Wave propagation in a micropolar elastic half-space, Acta Mech. 13 (1972), 11-20. MR 49\#6722. Zbl 255.73038.

[2] L. M. Brekhovskikh, Waves in Layered Media, Applied Mathematics and Mechanics, vol. 6, Academic Press, New York, 1960. MR 22\#3243.

[3] A. C. Eringen, Theory of micropolar elasticity, Fracture, vol. II, Academic Press, New York, 1968, pp. 621-729. Zbl 266.73004.

[4] A. C. Eringen and E. S. Suhubi, Nonlinear theory of simple micro-elastic solids. I, Internat. J. Engrg. Sci. 2 (1964), 189-203. MR 29\#6672. Zbl 138.21202.

[5] M. Fehler, Interaction of seismic waves with a viscous liquid layer, Bull. Seismol. Soc. Amer. 72 (1982), 55-72.

[6] R. D. Gautheir, Experimental Investigation on Micropolar Media, World Scientific, Singapore, 1982.

[7] R. D. Gautheir and W. E. Jashman, A quest for micropolar elastic constants, Trans. ASME J. Appl. Mech. 42 (1975), 369-373.

[8] R. Kumar and S. K. Tomar, Propagation of micropolar waves at boundary surface, Indian J. Pure Appl. Math. 27 (1996), no. 8, 821-835. Zbl 856.73015.

[9] J. W. Miles, Dispersive reflection at the interface between ideal and viscous media, J. Acoust. Soc. Amer. 26 (1954), 1015-1018. MR 16,973b.

[10] V. R. Parfitt and A. C. Eringen, Reflection of plane waves from the flat boundary of a micropolar elastic half-space, J. Acoust. Soc. Amer. 45 (1969), 1258-1272.

[11] B. Singh and R. Kumar, Reflection and refraction of plane waves at an interface between micropolar elastic solid and viscoelastic solid, Internat. J. Engrg. Sci. 36 (1998), 119135.

[12] E. S. Suhubi and A. C. Eringen, Nonlinear theory of micro-elastic solids. II, Internat. J. Engrg. Sci. 2 (1964), 389-404. MR 30\#751.

[13] S. K. Tomar and M. L. Gogna, Reflection and refraction of longitudinal wave at an interface between two micropolar elastic solids in welded contact, J. Acoust. Soc. Amer. 97 (1995), 827-830.

[14] S. K. Tomar and R. Kumar, Reflection and refraction of longitudinal displacement wave at a liquid-micropolar solid interface, Internat. J. Engrg. Sci. 33 (1995), no. 10, 15071515. Zbl 899.73114.

RAJNEesh Kumar: Department OF MATHematics, Kurukshetra University, KURUKSHETRA-136 119, HARYANA, INDIA

Sushil K. Tomar: Mathematics Department, Panjab University, Chandigarh-160 014, INDIA

E-mail address: sktomar@yahoo.com 


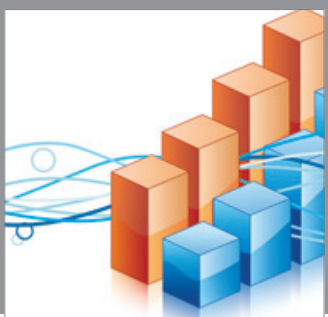

Advances in

Operations Research

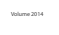

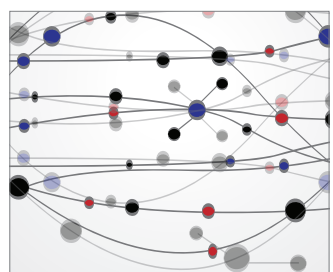

\section{The Scientific} World Journal
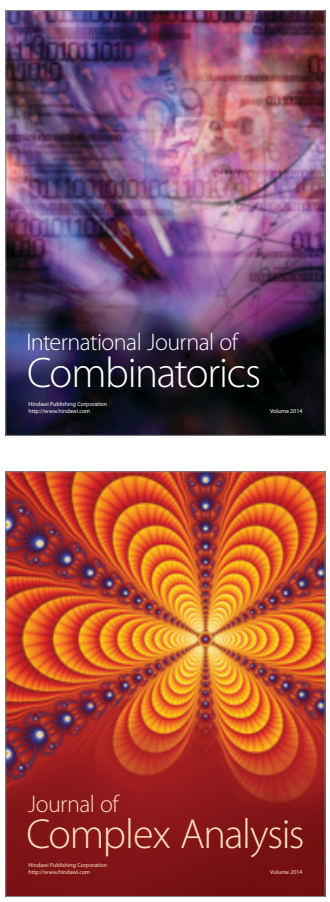

International Journal of

Mathematics and

Mathematical

Sciences
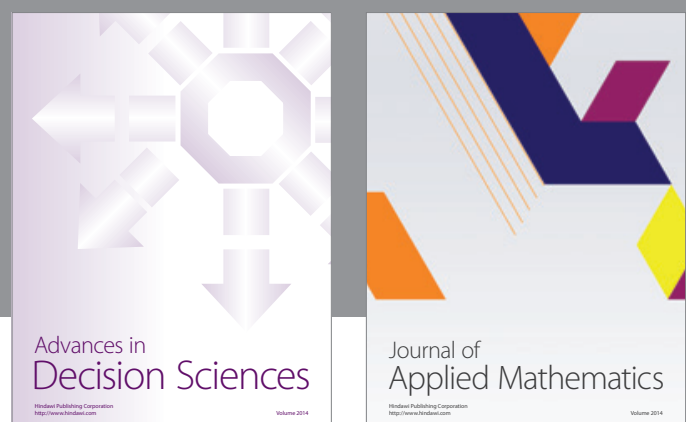

Journal of

Applied Mathematics
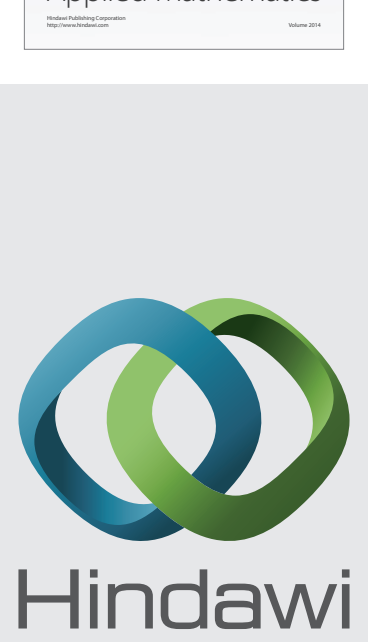

Submit your manuscripts at http://www.hindawi.com
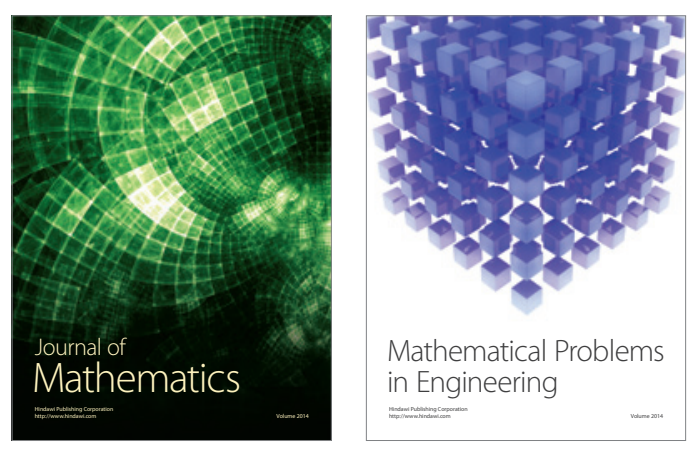

Mathematical Problems in Engineering
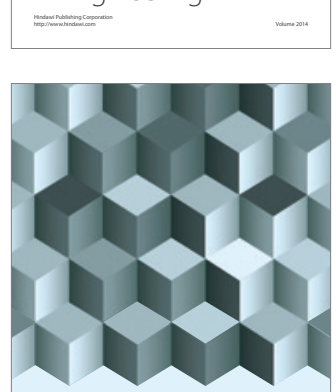

Journal of

Function Spaces
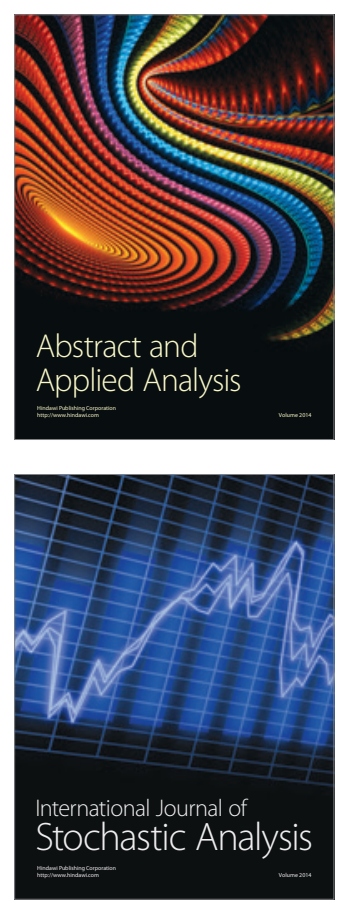

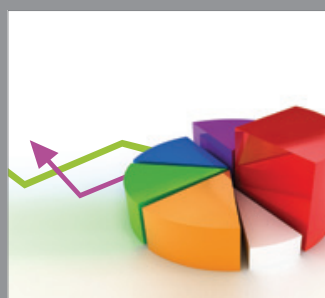

ournal of

Probability and Statistics

Promensencen
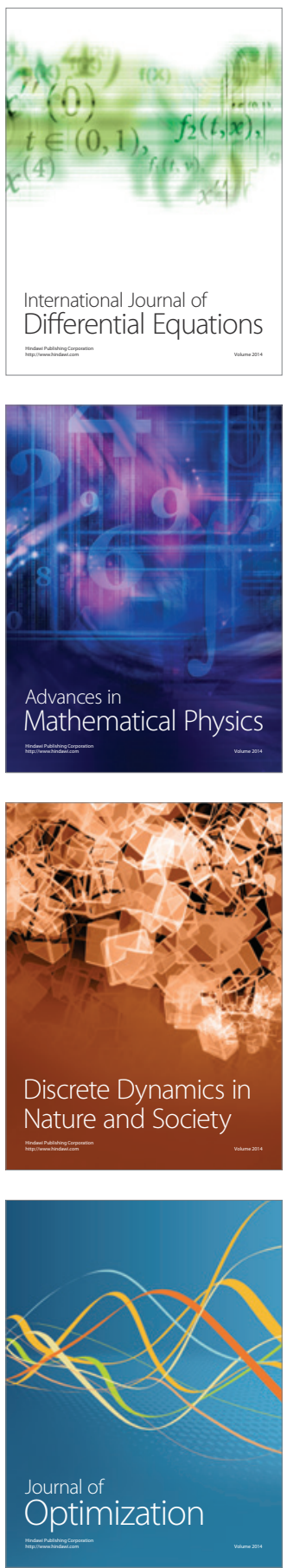